\title{
APPROXIMATE SOLUTION TO THE PROBLEM OF TORSION OF AN IN NITE ELASTIC ROD OF NORMAL CROSS-SECTION IN THE FORM OF A NEPHROID BY A BOUNDARY INTEGRAL METHOD.
}

\author{
S.M. SHESHTAWY* AND A. F. GHALEB ${ }^{* *}$ \\ Departments of Mathematics, Faculty of Science, Al-Azhar University*, Cairo University**
}

\begin{abstract}
The torsion of a long elastic bar possessing a normal cross-section bounded by a nephroid is considered by means of an expansion in polar harmonics, in conjunction with boundary collocation method and the boundary representation of harmonic functions.
\end{abstract}

Three types of nephroids are investigated and comparison is carried out between all three cases in what concerns the ed ciency of the used method.

The results are illustrated in three-dimensional plots of the unknown functions of practical interest.

Keywords: Theory of elasticity; plane elasticity; torsion of prismatic rods; boundary integral method; numerical solution; collocation method.

\section{INTRODUCTION}

The problem of torsion in the linear Theory of Elasticity has received considerable attention long ago as being a problem of practical interest. A large variety of torsion problems has been tackled using analytical techniques, mainly relying on results from the Theory of Potential. The basics of Torsion Theory may be found in all classical textbooks on the Theory of Elasticity [30, 64, 67, 69]. Due to the increasing mathematical dic culties encountered in the theoretical studies of torsion problems involving arbitrary boundary shapes, many purely numerical or semianalytical techniques have been used to solve such problems, including irregular boundaries, boundaries with corners and three-dimensional cases. [33, 35, 47, 48, 62, 70]. Christiansen [25] presents a review of integral equations to solve St. Venant $\mathrm{s}$ torsion problem. Jaswon and Ponter [41] develop a boundary integral equation to solve the torsion problem and present the solution to many geometries of the boundary, including boundaries with corner points like triangles and rectangles. Lo and Niedenfuhr [57] use an integral equation to solve the torsion problem, while Ponter [60] deals with the inhomogeneous torsion problem. Bhargava and Puranik $[14,15]$ investigate the torsion of prismatic bars with multiconnected normal cross-section by boundary integrals. Boundary integral equations are also used in [75] to solve torsion problems. Torsion of bars with boundaries having corner points is also treated by Kolodziej and Fraska [50] and for special types of boundaries in [13]. Torsion of composite bars by boundary element method was treated in [46]. Holl and Anderson [39] studied the limits of deviation of the approximate solution of a torsion problem from the exact solution.

The numerical methods are considered to be powerful and necessary tools for analyzing a wide range of engineering applications. The tremendous development of computer technology in the past few decades has added to the importance of the numerical methods by allowing dic cult numerical tasks and time-consuming calculations to be implemented relatively easily. The level of accuracy was raised consequently. The use of mesh-based methods, such as nite dierences, nite elements or nite volumes methods have been widely investigated. In all methods, the natural boundary of the body is usually replaced by a polygonal shape which involves a multitude of corner points and necessarily adds or deletes parts to the real region occupied by the body. This, in turn, necessitates the application of boundary conditions on arti cial boundaries, a fact that introduces additional inaccuracies into the solution. Minimizing the error requires large computing times. One way for the e/orts to overcome these problems was the self-adjusting mesh generation at each iteration level and tests for minimal error. This, however, is a dic cult task which requires advanced methods. The 
second way to overcome such problems and remove many of the disadvantages of the numerical techniques was the use of alternative, semianalytical treatments. An increasing interest in developing the so-called meshless methods has been witnessed in the past few decades. Such approaches are usually classi ed under the general title of Boundary Integral Methods. They have the advantage of reducing the volume of calculations by considering, at one stage, only the boundary values of the unknown functions and then using them to nd the complete solution in the bulk. In addition, these procedures deal exclusively with the real boundary of the medium, restricted, though, to certain regularity conditions and need not introduce arti cial boundaries. An extensive account of boundary integral equation methods in potential theory and in elastostatics may be found in [42]. Boundary methods were also treated by Herrera and Gourgen [38]. Boundary integral equations to solve Laplace s equation in two-dimensions were treated in [16, 17]. Treatment of boundary singularities is dealt with in [65]. Constanda [27, 28] investigates the use of boundary integral equations in plane elasticity.

Meshless methods for solving boundary value problems have been extensively popularized owing to their exibility in engineering applications, especially for problems with discontinuities and because of high accuracy of the computed results. They focus only on the points, instead of the mesh elements as in the conventional nite element method. A meshfree method does not require a mesh to discretize the domain of the problem under consideration, and the approximate solution is constructed entirely based on a set of scattered nodes. The meshless methods often lead to boundary integral equations [10, $18,53]$. This is one of the strong points of these methods, since the integral equations have been a focus of interest long ago. Several theorems on existence, uniqueness and stability of solutions of integral equations were established. In recent years, there has been a growing interest in the integro-di/erential equations. A review of boundary integral equations is presented in [59]. Several domain type meshfree methods, among which the meshless Petrov-Galerkin method [9,
$11,12]$ have been proposed. Applications to potential problems are investigated in [71]. These methods achieved remarkable progress in solving a wide range of static and dynamic problems. In concept, any consistent set of boundary conditions will yield a solution within the framework of meshless methods. Practically, boundary conditions may be very cumbersome and yield singularities in the integral equations which cause numerical dic culties. This is the case in boundary-value problems with mixed boundary conditions. The use of boundary integrals to solve concrete problems of the Theory of Elasticity and in other special contexts may be found in [2, $3,32,34,63,66]$.

Trettz in 1926 [68] introduced a method, later named the Tretz method, for solving boundaryvalue problems. It soon became quite popular. In this method, the solution to the considered problem is expressed as a superposition of functions satisfying the governing equation. Various versions of the Tretz method, e.g., direct and indirect formulations have been developed. The unknown coe $\phi$ cients are then determined by matching the boundary condition. A review of Tretz method may be found in [49] and contributions on this topic in $[31,55,72,73]$. The relation between the method and the boundary integral equations is revealed in [40]. Investigation of some problems by Tre/tz method is carried out in [1]. A comparison between Tretz method and other boundary methods may be found in [56]. Comparison with nite element method was investigated in [51] for some two-dimensional problems. This method was also applied in conjunction with other methods in solving plane problems of static Elasticity, Thermoelasticity and Thermo-magnetoelasticity [4]-[7]. The analysis of Tretz method in what concerns the completeness of the used expansion basis was undertaken by Jirousek and Wr blewski [43], and by Herrera [36, 37]. The collocation method collocation method in conjunction with a boundary Fourier expansion was treated in $[74,8]$.

Trettz method and MFS are both mesh reduction methods. Boundary collocation techniques are among meshless methods. They are getting much attention for the solution to various partial dierential equations which are useful in many 
practical applications. The history of boundary elements has been reviewed in a recent paper by Cheng and Cheng [23]. The use of these methods involves boundary discretization and hence provides a reduction in the dimensionality of the problem.This, in turn, increases the ed ciency of the solution method considerably and also provides a direct and more accurate estimate of the gradients at the boundary compared to other methods. Although boundary methods are most suitable for linear problems (constant physical parameters, geometric linearity, etc.), they can be useful for the study of nonlinear problems as well [24].

In the potential theory, it is well known that the method of fundamental solutions (MFS) can solve potential problems e ciently. Extensive applications in solving a broad range of problems have been investigated $[29,44,45,58,61]$. The MFS can be viewed as an indirect boundary element method (BEM) with concentrated sources instead of boundary distributions. The initial idea is to approximate the solution of Laplace $s$ equation through a linear combination of fundamental solutions with sources located outside the domain of the problem. Moreover, it has certain advantages over BEM, e.g., no singularity and no boundary integral. However, ill-posed behavior is inherent in the regular formulation. Mathematical studies on the MFS have been investigated by some researchers. Bogomolny [19] studied the stability and error bound of MFS. Christiansen and Hansen [26] used the e/ective condition number to carry out an error analysis of some collocation methods. They found that the condition number of the coed cient $\mathrm{s}$ matrix of MFS is much worse than that of the Tre/tz method. Li et al. [54] investigate special approximation boundary methods for Laplace s equation with a view towards boundary singularities.

The boundary element method (BEM) is a numerical computational method of solving linear partial di/erential equations which have been formulated as integral equations, i.e. in boundary integral form. It can be applied in many areas of engineering and science, including uid mechanics, acoustics, electromagnetics and fracture mechanics. Interested reader is referred to the work of Brebbia [20] and Brebbia and Dominguez [21].

The present work investigates the torsion of a long elastic bar possessing a normal crosssection bounded by a nephroid, by means of moments applied to the bases, the lateral surface being stress free. The warping function is expressed as an expansion in polar harmonics and the coe $\varnothing$ cients are determined by Boundary Collocation Method. The resulting system of linear algebraic equations is tested each time for the determinant and the 2-norm of the matrix of coec cients to avoid ill-posedness. The obtained solution is then used to evaluate the maximum error in satisfying the main theorem on the boundary representation of harmonic functions in discretized form and after regularization. This is taken as a measure of the ed ciency of the proposed scheme. A table shows the maximum error against the number of nodes used in the discretized form of the boundary integral representation. Three types of nephroids are investigated and comparison is carried out between the three cases in what concerns the e ciency of the used method and the distribution of stresses in the bulk. The results are illustrated in three-dimensional plots of the unknown functions of practical interest. It is shown that the convergence of the procedure becomes weaker as the lobes of the nephroid are more pronounced.

\section{Problem formulation and basic equations}

Let the bases of the homogeneous isotropic prismatic elastic bar be acted upon by forces that reduce to twisting couples. It will be assumed that the body forces are absent and that the lateral surface of the bar is free from external forces. The normal cross-section of the bar is a twodimensional, simply connected region bounded by a closed contour $S$. A system of orthogonal Cartesian coordinates $(x ; y ; z)$ is used in the plane of , with origin at $O 2$, the $z$-axis being parallel to the generators the lateral surface.

For the application, the normal cross-section is in the form of a nephroid with major and minor axes lengths $\frac{2 h^{2}}{b}, \frac{2 h^{2}}{a}$ respectively. The parameter $a$ is taken as a representative length for the problem. Its value is taken equal to 1 for 
convenience. The parametric equations of the boundary in dimensionless form read

$$
\begin{aligned}
x(\theta) & =c_{1} \frac{\cos \theta}{\cos ^{2} \theta+\epsilon^{2} \sin ^{2} \theta}, \quad y(\theta)=c_{1} \frac{\epsilon \sin \theta}{\cos ^{2} \theta+\epsilon^{2} \sin ^{2} \theta}, \\
0 & \leq \theta \leq 2 \pi, \quad(1)
\end{aligned}
$$

where $\theta$ is the polar angle in the plane of the cross-section de ned in the usual way, and

$c_{1}=\frac{h^{2}}{a}, \epsilon=\frac{b}{a}$. Three cases of interest are represented on Figs. $(1,2,2)$. The following values

of the parameters are used: $h=0: 45$ for Fig. (1); $h=0: 35$ for Fig.(2) and $h=0: 35$ for

Fig.(2).

Let be the unit vector tangent to $S$ at a general point $Q$ in the sense of increase of and $\mathbf{n}$ the unit outward normal to $S$ at $Q$. The ordered pair fn; $\mathrm{g}$ forms a right-handed system. It can be shown

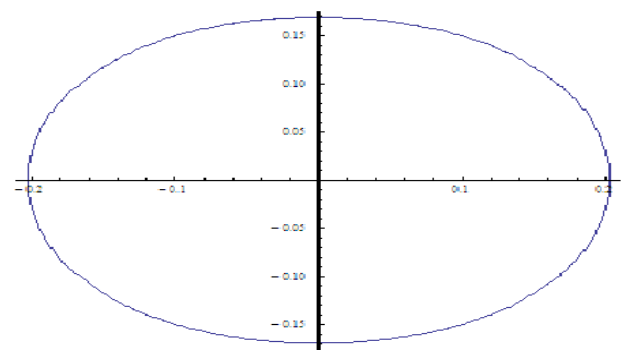

Figure 1: Case I: $a=1.0, b=1.2$

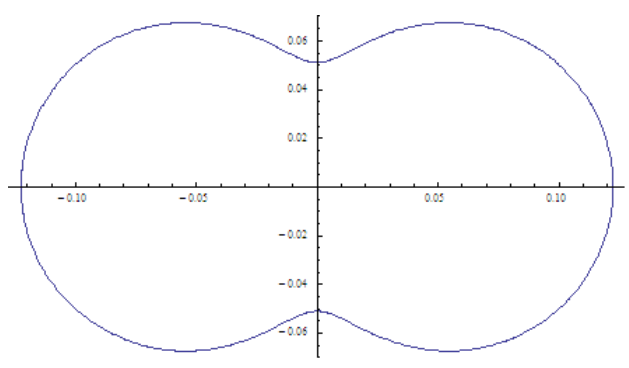

Figure 2: Case II: $a=1.0, b=2.4$

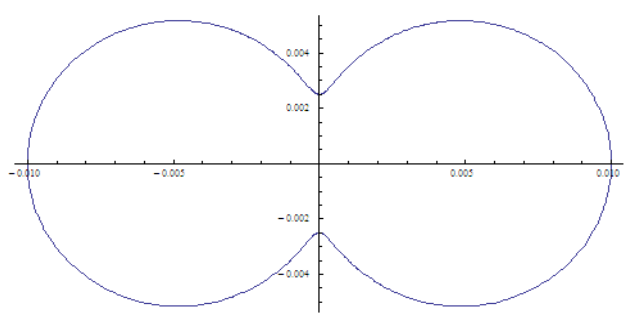

Case III: $a=1.0, b=3.5$

$$
\text { that }\left(\frac{\dot{x}(\theta)}{\sqrt{\varpi}}, \frac{\dot{y}(\theta)}{\sqrt{\varpi}}\right), \mathbf{n}=\left(\frac{\dot{y}(\theta)}{\sqrt{\varpi}},-\frac{\dot{x}(\theta)}{\sqrt{\varpi}}\right),
$$

the dot over a symbol denotes dierentiation w.r.t. and $\varpi=(\dot{x})^{2}+(\dot{\bullet})^{2}$.

If the natural parametric representation for the boundary $S$ is used, i.e. if the running parameter on the boundary is the arc length, then

$$
\$=1 \text { : }
$$

It is worth noting that $S$ is su $\varnothing$ ciently smooth, as this is an important factor for an e $\varnothing$ cient application of the proposed method. Torsion of cylinders with cross-section having angular boundary points will be considered in separately.

\section{Torsion of prismatic rods}

The solution of the formulated problem in terms of displacements is sought by Saint Venant in the form

$$
U=-\tau y z, \quad V=\tau z x, \quad W=\tau \phi(x, y),
$$

where is a constant called the degree of twist and $(x ; y)$ is a function to be determined. It is known as Saint Venant $s$ torsion function or warping function. Displacements (4) show that the cross-sections do not remain plane but warp. Moreover, all sections warp identically. The only cross-section that does not warp was known to be the circular one. Later on, Chen [22] has shown that elliptical cross sections may also exhibit zero warping under some condition: The cylinder needs to be rectilinearly orthotropic in which the ratio of two associated shear rigidities equals the square of the aspect ratio of the ellipse. Physically, this means that the elastic orthotropy of the shaft can serve to compensate the geometric deviation from a circular cross-section to an elliptical one. As Chen points out, the idea can be further generalized to show that the zero warping property also holds for a number of composite cylinders consisting of an elliptical core or cavity coated with many similarly elliptical layers of dierent materials.

$$
\sigma_{z x}=\mu \tau\left(\frac{\partial \phi}{\partial x}-y\right), \quad \sigma_{z y}=\mu \tau\left(\frac{\partial \phi}{\partial y}+x\right),(5)
$$

The stress tensor components corresponding to the displacements in (4) are expressed as: 
and

$$
\sigma_{x x}=\sigma_{y y}=\sigma_{z z}=\sigma_{x y}=0 .
$$

Hereis one of two Lam $\varnothing$ coed cients characterizing the elastic material.

In the absence of body forces, the warping function is harmonic in the variables $x, y$ in the region $S$ occupied by the cross-section of the body: 2

$$
\nabla^{2} \phi=0 .
$$

Let $(x ; y)$ be the harmonic conjugate to the function $(x ; y)$. The Cauchy-Riemann conditions are: $\frac{\partial \phi}{\partial x}=\frac{\partial \psi}{\partial y}, \quad \frac{\partial \phi}{\partial y}=-\frac{\partial \psi}{\partial x}$,

The boundary condition satis ed by the function $(x ; y)$ is:

$$
\left.\psi\right|_{L}=\frac{1}{2}\left(x^{2}+y^{2}\right)+C,
$$

where $C$ is an arbitrary constant, while the components of the stress tensor are obtained from as:

$\sigma_{z x}=\mu \tau\left(\frac{\partial \psi}{\partial y}-y\right), \quad \sigma_{z y}=-\mu \tau\left(\frac{\partial \psi}{\partial x}-x\right)$.

It is well seen from the above formulas that the solution of the problem will not change if a constant is added to the function $(x ; y)$. Consequently, the determination of this function is reduced to solving the Dirichlet problem for Laplace $\mathrm{s}$ equation.

The shearing stresses applied to the crosssection reduce to a couple of moment

$$
M=\int_{\Gamma}\left(x \sigma_{z y}-y \sigma_{z x}\right) d \Gamma .
$$

Inserting into this formula the values of $z y^{\prime} z x$ calculated from formulas (5), one nally obtains

$$
M=D \tau \text {. }
$$

In this formula

$$
D=\mu \int_{\Gamma}\left(x^{2}+y^{2}+x \frac{\partial \phi}{\partial y}-y \frac{\partial \phi}{\partial x}\right) d \Gamma
$$

is the torsional rigidity. If $M_{t}$ is the twisting moment or torque at a base, then the equilibrium condition yields $M_{t}=M=D$; from which

$$
\tau=\frac{M_{t}}{D} \text {. }
$$

Boundary integral representation of harmonic functions

Let $F$ be a harmonic in . One uses the wellknown integral representation from Potential Theory for $F$ at an arbitrary eld point $(x ; y)$ in in terms of the boundary values of the function $F$ and it s complex conjugate $F^{c}$ in the form

$F(x, y)=\frac{1}{2 \pi} \oint_{S}\left[F\left(s^{\prime}\right) \frac{\partial}{\partial n^{\prime}} \ln R+F^{c}\left(s^{\prime}\right) \frac{\partial}{\partial s^{\prime}} \ln R\right] d s^{\prime}$,

where $R$ is the distance between the point $(x ; y)$ in and the current integration point $\left(x\left(^{0}\right) ; y(\right.$ $\left.{ }^{0}\right)$ ) on $S$.

The representation of the conjugatate function is given by

$F^{c}(x, y)=\frac{: 1}{2 \pi} \oint\left[F^{c}\left(s^{\prime}\right) \frac{\partial}{\partial n^{\prime}} \ln R-F\left(s^{\prime}\right) \frac{\partial}{\partial s^{\prime}} \ln R\right] d s^{\prime}$.

the integral representations $(12,13)$ for the harmonic function $F$; $F^{c}$ replace the usual Cauchy-Riemann conditions.

When the point $(x ; y)$ tends to a boundary point $\left(x\left(s^{\prime}\right), y\left(s^{\prime}\right)\right)$ relation (12) yields:

$$
F(s)=\frac{1}{\pi} \oint_{S}\left[F\left(s^{\prime}\right) \frac{\partial}{\partial n^{\prime}} \ln R+F^{c}\left(s^{\prime}\right) \frac{\partial}{\partial s^{\prime}} \ln R\right] d s^{\prime},
$$

Replacing $\frac{\partial}{\partial n^{\prime}} \ln R$ by $\frac{\partial}{\partial s^{\prime}}$ in $(12,13)$ and their boundary version (14), where is the harmonic conjugate of $\ln R$, it is readily seen that these integral relations are invariant under the transformation of parameter from the arc length $s$ to any other suitable parameter. This property makes the method more exible. In view of the integral representations $(12,13)$, it is su $\varnothing$ cient for the complete solution of the mechanical problem in the region to determine the boundary values of the harmonic function $F$, as well as those of the harmonic conjugate. This requires two independent relations in these unknowns, obtained from (14) written for. $F$ and it $\mathrm{s}$ conjugate. For the application, it is more convenient to use the following form of the integral representation for any harmonic function $F$ : 
Numerical scheme for the solution

In this section the dierential and integral operators appearing in the equations are to be discretized as usual and the problem of determination of the boundary values of the unknown functions reduces to nding the solution of a linear system of algebraic equations. The full solution is then obtained by numerical quadrature according to $(13,14,15)$.

The discretization procedure

The contour of integration $S$ will be divided into a nite number $p$ of segments $G_{1} ; G_{2} ;: ; ; G_{p}$ of respective lengths $S_{1} ; S_{2} ;: ; ; S_{p}$ not necessarily equal. For convenience only, the value of $p$ will be chosen so that $(p 1)$ is divisible by 4 . Let $Q_{i}$ $=\left(x_{i} y_{i}\right)$ be the midpoint of the segment $G_{i}(i=$ $1 ; 2 ;: ; ; p)$ with corresponding value $S_{i}$ of the parameter $S$ (the points $Q_{i}$ may in fact be arbitrarily chosen in the intervals $G_{i}$ ). The lengths of these segments are chosen small enough so that the value of any continuous function $f(s)$ de ned on $S$ may be approximated on the segment $G_{i}$ by it s value at the point $Q_{i}$, denoted $f_{i}$ : The point $Q_{0}$ appearing in the above formulation is identi ed with any one of the points $Q_{i}$, say $Q_{1}$ : Any contour integration on $S$ will be replaced, as an approximation, by the Riemann sum

$$
\oint f(s) d s=\sum_{i=1}^{p} f_{i} \Delta S_{i},
$$

The total number of unknowns on the boundary is $2 p$ representing the values of the harmonic functions ; ${ }^{c}$, where ${ }^{c}$ is the complex conjugate of, at the points $Q_{i}(i=1 ; 2 ; 3 ;: ; ; p)$. These unknowns will be considered as the components of a $2 p$-dimensional vector $X$ with components $\left(X_{1} ; X_{2} ; \ldots ; X_{2 p}\right)$ de ned according to the rule:

$$
X_{1}=\psi_{1}, \ldots, X_{p}=\psi_{p}, \quad X_{p+1}=\psi_{1}^{c}, \ldots, X_{2 p}=\psi_{p}^{c}
$$

This procedure for the unknowns $\left(X_{1} ; X_{2} ;: ; ; X_{2 p}\right)$ yields an overdetermined system of linear algebraic equations which can be written in the matricial form

$$
A X=B \text {; }
$$

the general elements $\left(A_{m n}\right)$ and $\left(B_{m}\right)$ of matrix $A$ and vector $B$ are determined in the following subsection. tions

Discretization of the Cauchy-Riemann condi-
This concerns the discretization of equation (15) and provides $2 p$ rows in the coe cient $\mathrm{s}$ matrix. The discretization of this equation leads to the following $p$ algebraic equations.

$$
\begin{array}{r}
\psi_{i}=\frac{1}{\pi} \sum_{j=1}^{p}\left[\psi_{j} \frac{\partial}{\partial n_{j}} \ln R_{i j}+\left(\psi_{j}^{c}-\psi_{i}^{c}\right) \frac{\partial}{\partial s_{j}} \ln R_{i j}\right] \Delta s_{j} \\
i=1,2, \ldots \ldots \ldots . ., p
\end{array}
$$

where $\frac{\partial}{\partial n_{j}}$ and $\frac{\partial}{\partial s_{j}}$ denote respectively the directed derivatives along the normal and the

tangent to the contour $S$ at the point $Q_{j}$ and $R_{i j}$ is the distance between the two points $Q_{i}$ and $Q_{j}$. Clearly, when $i=j$ there will be a singularity in the corresponding summation term and this requires a special treatment to remove it. For convenience, one sets

$$
\begin{gathered}
W_{i j}=\left(\frac{\partial}{\partial n_{j}} \ln R_{i j}\right) \Delta s_{j}, \quad Z_{i j}=\left(\frac{\partial}{\partial s_{j}} \ln R_{i j}\right) \Delta s_{j}, \\
i, j=1,2, \ldots, p
\end{gathered}
$$

The following formulas may be easily veri ed [7]

$$
\begin{gathered}
W_{i j}=\frac{\dot{y}_{j}\left(x_{j}-x_{i}\right)-\dot{x}_{j}\left(y_{j}-y_{i}\right)}{,} R_{i j}^{2} s_{j}, \quad i \neq j, \\
W_{i i}=\frac{-1}{2} \frac{\ddot{x}_{i} \dot{y}_{i}-\ddot{y}_{i} \dot{x}_{i}}{\left(\dot{x}_{i}\right)^{2}+\left(\dot{y}_{i}\right)^{2}} \Delta s_{i},
\end{gathered}
$$

Formulas $(19,20,21)$, to our belief, are more accurate than those usually used in the boundary integral equation methods existing in the literature. These methods replace the actual boundary by a polygon over which the line integrals are performed, while the present formulation takes into account the shape of the actual boundary through the rst and the second derivatives of the functions $x(s) ; y(s)$. The quantities $x_{i} y_{i}$ as well as their derivatives w.r.to the parameter $s$ are known once the contour has been precised. Formula (21) requires the existence and continuity of the second derivatives of the functions, i.e. the closed boundary $S$ must belong to class $C^{2}$ at least. In fact, this is the condition imposed by Constanda when investigating the uniqueness and existence of the solution by the Boundary Integral Method .

$$
Z_{i j}=\frac{\dot{x}_{j}\left(x_{j}-x_{i}\right)+\dot{y}_{j}\left(y_{j}-y_{i}\right)}{R_{i j}^{2}} \Delta s_{j}, \quad i \neq j
$$


For the part involving $Z_{i j}$ one has [7]

Calculation of the harmonic functions at internal points

Having determined the boundary values of the harmonic functions on the boundary by using formulae for the dimensionless function ${ }_{i}$ :

$$
\begin{gathered}
\bar{\psi}_{i}=\frac{\psi_{i}}{a^{2}}=\frac{1}{2 a^{2}}\left(x_{i}^{2}+y_{i}^{2}\right)+c_{1}, \quad(22) \\
\left.\bar{\psi}=\frac{\psi}{a^{2}}=a_{0}+\sum_{n=1}\left(a_{n} \cos (n x)+b_{n} \sin (n x)\right] \frac{\exp (n y)}{T \exp (n c)}+\left[c_{n} \cos (n x)+d_{n} \sin (n x)\right] \exp (-n y)\right) .
\end{gathered}
$$

For best results, one takes $T=0.1$ in case (I), $T=0.01$ in case (II) and $T=0.001$ in case (III), and

The Cauchy-Riemann conditions yield:

$$
N=\frac{p-1}{4}, \quad c=\frac{h^{2}}{b}
$$

$$
\left.\bar{\psi}^{c}=\frac{\psi^{c}}{a^{2}}=k+\sum_{n=1}^{N}\left(-a_{n} \sin (n x)+b_{n} \cos (n x)\right] \frac{\exp [n y]}{T \exp [n c]}+\left[c_{n} \sin (n x)-d_{n} \cos (n x)\right] \exp (-n y)\right) .
$$

One takes $k=0: 01$ in case (I) and $k=0: 005$ in case (II) and $k=0: 5$ in case (III). The additional constant has no physical implications, its value amounts to xing the value of the harmonic conjugate function at an arbitrarily chosen point of the domain. Numerical experiments, however, have indicated that the value of this constant may strongly a/ect the calculations. For best results, one chooses $k$ as follows: $k=0: 1$ in case (I), $k=0: 3$ in case (II) and $k=0: 6$ in case (III).

The Boundary Collocation Method is then applied to determine the coed cients in these expansions in terms of the previously obtained boundary values of these functions. The resulting system of linear algebraic equations was solved by Least Squares method. Each time, the determinant and the 2-norm of the coe $\phi$ cient $\mathrm{s}$ matrix was calculated to avoid ill-posedness of the system, the results are shown on the Table below. Also, the solution was substituted again into the equations in order to con rm its exactitude.
In the end, the obtained expansions were substituted again into the boundary integral representation of harmonic functions in discretized form and after regularization. The maximum error in satisfying these conditions is noted in the Table below. It will serve as a measure of the e $\varnothing$ ciency of the used procedure. The stress components may now be calculated from (10):

\section{Numerical results and discussion}

The value of $p$ was taken such that $(p 1)$ be divisible by 4 for convenience. In fact, only 5 nodes were required to obtain the coed cients of the expansion. Further increase of the number of nodes up to 201 nodes was used to verify the boundary integral representation of harmonic functions. The following Table shows the determinant and the 2-norm of the matrix of coe $\phi$ cients in determining the coe $\phi$ cients of the expansion and the value of the twisting moment for the three cases under consideration by 5 nodes.

$$
\begin{aligned}
\bar{\sigma}_{z y}= & \frac{\sigma_{z y}}{\mu \tau a}=\sum_{n=1}^{N}\left(\left[a_{n} \sin (n x)-b_{n} \cos (n x)\right] \frac{n \exp (n y)}{T \exp (n c)}+\right. \\
& {\left.\left[-c_{n} \sin (n x)+d_{n} \cos (n x)\right][n \exp (-n y)]\right)+x, } \\
\bar{\sigma}_{z x}= & \frac{\sigma_{z x}}{\mu \tau a}=\sum_{n=1}^{N}\left(\left[a_{n} \cos (n x)+b_{n} \sin (n x)\right] \frac{n \exp (n y)}{T \exp [(n c)]}+\right. \\
& {\left.\left[c_{n} \cos (n x)+d_{n} \sin (n x)\right][-n \exp (-n y)]\right)-y . }
\end{aligned}
$$




\begin{tabular}{|c|c|c|c|}
\hline & case (I) & case (II) & case (III) \\
\hline det & -0.0376 & -0.0086 & -0.0917 \\
\hline 2-norm & 19.30 & 212.7 & 2159.5 \\
\hline M & 1.57 & 3.86 & 0.8161 \\
\hline
\end{tabular}

Ultimately, the basic harmonic function and its conjugate could be plotted in 3-dimensions in the cross-section domain (Figs.(6)-Fig.(6)), as well as the stress components acting on the cross-section (Figs.(6)-Fig.(6)). The results are shown below, where the domain of the solution (the normal cross-section of the cylinder) has also been represented together with the plotted functions for convenience.

The following Table shows the error in satisfying the boundary integral representation of harmonic functions against the number of nodes.

\begin{tabular}{|c|c|c|c|}
\hline$p$ & case (I) & case (II) & case (III) \\
\hline 5 & $0: 00998$ & $0: 00893$ & $0: 00954$ \\
\hline 73 & $0: 00640$ & $0: 00201$ & $\mathbf{0 : 5 6 9 2 1}$ \\
\hline 121 & $0: 00039$ & $0: 00095$ & $0: 00694$ \\
\hline 161 & $0: 00029$ & $0: 00074$ & $0: 00130$ \\
\hline 201 & $0: 00023$ & $0: 00059$ & $0: 00099$ \\
\hline
\end{tabular}

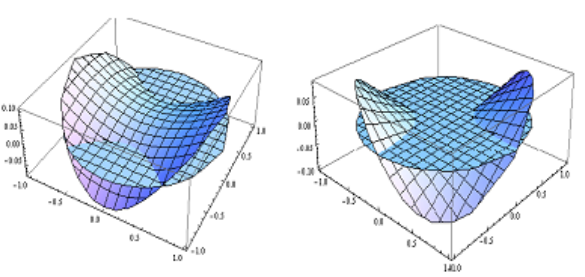

Function $\bar{\psi}$ (left) and $\bar{\psi}^{c}=-\bar{\phi}$ (right) for case (I).
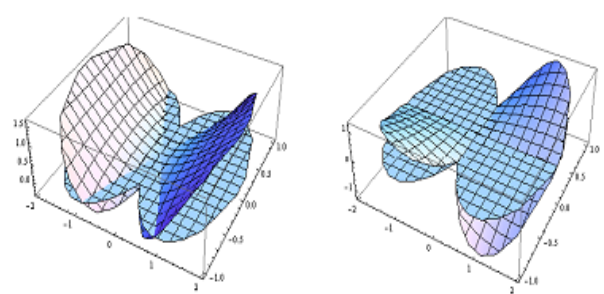

Function $\bar{\psi}$ (left) and $\bar{\psi}^{c}=-\bar{\phi}$ (right) for case (II).
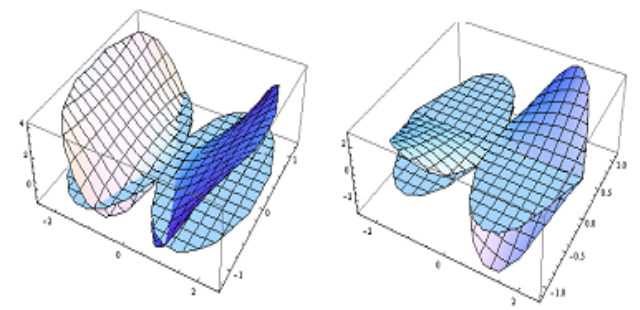

Function $\bar{\psi}$ (left) and $\bar{\psi}^{c}=-\bar{\phi}$ (right) for case (III).

It is readily seen that the warping function, as well as the stresses, for the ellipse-like contour are qualitatively dierent from those of the contour with two lobes. This is compatible with the fact that the ellipse-like contours are the only convex nephroids. The symmetry properties of the stress components are obvious. It is also noticed that the 2-norm of the matrix grows larger as the lobes of the boundary become more pronounced, so one expects the ed ciency of the method to deteriorate in that case. This is accompanied by a growth of the stresses and a slower convergence of the maximum error to zero.

\section{REFERENCES}

M. S. Abou-Dina, Implementation of Tretz s method for the solution of some elliptic boundary-value problems, Appl. Math. Comp., (2002); 127:125-147.

M. S. Abou-Dina, A. A. Ashour, A general method for evaluating the current system and its magnetic eld of a plane current sheet, uniform except for a certain area of dierent uniform conductivity, with results for a square area, Il Nuovo Cimento (1989); 12 C: 5, 523-540.

M. S. Abou-Dina, A. A. Ashour, Exact solutions for certain two-dimensional problems of induction in thin nonuniform sheets, Journal of Geophysical Research (1990); 95:547553.

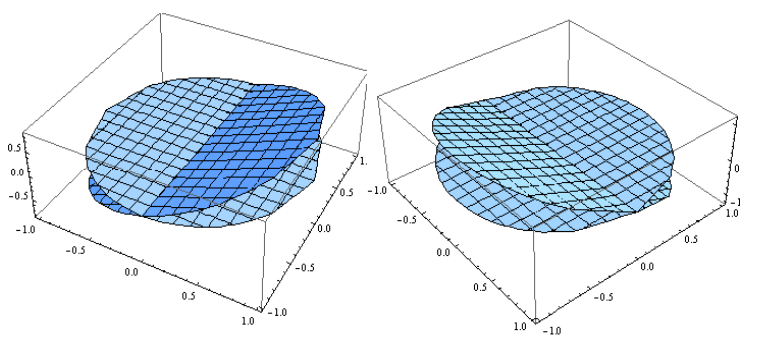

Stresses $\sigma_{y z}$ (left) and $\sigma_{x z}$ (right) in case (I).

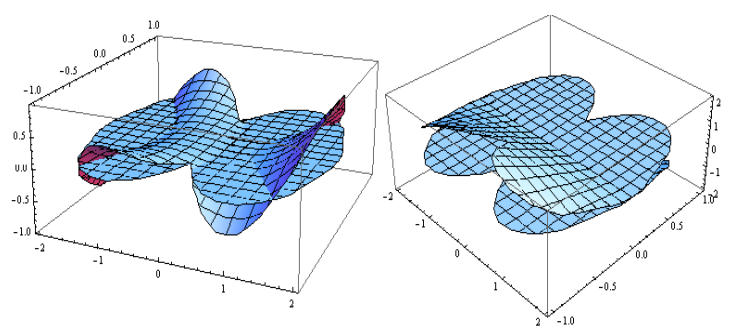

Stresses $\sigma_{y z}$ (left) and $\sigma_{x z}$ (right) in case (II).

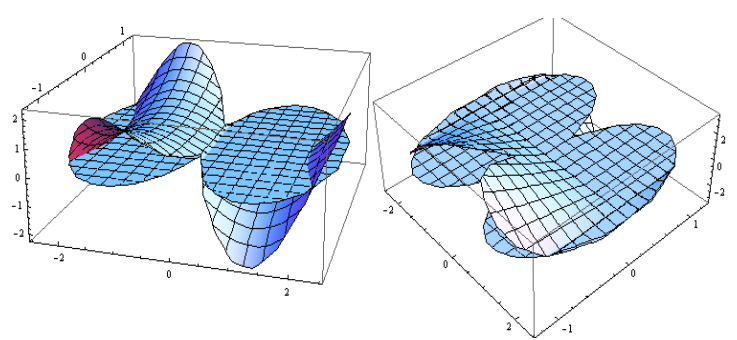

Stresses $\sigma_{y z}$ (left) and $\sigma_{x z}$ (right) in case (III). 
M. S. Abou-Dina, A. F. Ghaleb, On the Boundary Integral Formulation of the Plane Theory of Elasticity with Applications (analytical aspects), J. Comp. and Appl. Math. (1999); 106 (1): 55-70.

M. S. Abou-Dina, A. F. Ghaleb, On the boundary integral formulation of the plane theory of Thermo-magnetoelasticity, In Studies in Applied Electromagnetics and Mechanics , vol. 19, Mechanics of Electromagnetic Materials and Structures, Eds. J. S. Yang and G. A. Maugin, pp. 77-98, IOS Press, Amsterdam Berlin Oxford Tokyo Washington (2000). Int. J. Appl. Electromagn. and Mech. (2000); 11: 185-201.

M. S. Abou-Dina, A. F. Ghaleb, On the boundary integral formulation of the plane Theory of Thermoelasticity, J. Thermal Stresses (2002); 25 (1): 1-29.

M.S. Abou-Dina, A.F. Ghaleb, On the boundary integral formulation of the plane theory of elasticity (computational aspects), Journal of Computational and Applied Mathematics (2003); 159: 285317.

M. S. Abou-Dina, A. F. Ghaleb, A variant of Tretz s method by boundary Fourier expansion for solving regular and singular plane boundary-value problems. J. Comp. Appl. Math. (C.A.M.) (2004); 167: 363-387.

S. N. Atluri, S. Shen, The meshless local Petrov-Galerkin (MLPG) method: a simple and less-costly alternative to the nite element and boundary element methods. CMES: Comp. Model. Engng. Sci.(2002); 3:11-51.

S. N. Atluri, J. Sladek, V. Sladek, T. Zhu, The local boundary integral equation (LBIE) and it s meshless implementation for linear elasticity, Computational Mechanics (2000); 25:180-198.

S. N. Atluri, T.-L. Zhu, The meshless local Petrov-Galerkin (MLPG) approach for solving problems in plasto-ptatics, Computational Mechanics (2000); 25: 169-179.

S. N. Atluri, T. L. Zhu, The meshless local Petrov-Galerkin (MLPG) approach for solving problems in elasto-statics, J. Comp. Mech. (2002); 25: 169179.

D. R. Axelrad, An approximate solution for the torsional properties of cylindrical shafts with multi-symmetrical curved boundaries, ZAMP (1962); 13 (2): 105-117.

R. D. Bhargava, S. S. Puranik, Torsion problem for elastic cylinder with holes, Computer Methods in Applied Mechanics and Engineering (1980); 21:63-74.

R. D. Bhargava, S. S. Puranik, Torsion problem for elastic cylinder with inserts and holes, Computer Methods in Applied Mechanics and Engineering (1980); 23: 281291.

R. D. Bhargava, R. S. Saxena, Integral equation method of solving two-dimensional Laplace equation-I, Ind. J. Pure Appl. Math. (1970); 1: 552-564.

R. D. Bhargava, R. S. Saxena, Integral equation method of solving two-dimensional Laplace equation-II, Ind. J. Pure Appl. Math. (1971); 2: 246-256.

A. Bodin, J. Ma, X.J. Xin, P. Krishnaswami, A meshless integral method based on regularized boundary integral equation, Comput. Methods Appl. Mech. Engrg. (2006); 195: 62586286.

A. Bogomolny, Fundamental solutions method for elliptic boundary-value problems, SIAM Journal on Numerical
Analysis (1985); 22(4): 644669.

C. A. Brebbia, Recent advances in boundary element method, Pentech Press, London

(1978).

C. A. Brebbia, J. Dominguez, Boundary element methods for potential problems, Applied Mathematical Modelling (1977); 1: 372-378.

T. Chen, A homogeneous elliptical shaft may not warp under torsion, Acta Mechanica (2004); 169 (1-4): 221 224.

A. H.-D. Cheng, D. T. Cheng, Heritage and early development of boundary element, Eng. Anal. Bound. Elem. (2005); 29: 268302.

W. Chen, M. Tanaka, A meshless, integration-free, and boundary-only RBF technique, Computers and Mathematics with Applications (2002); 43: 379-391.

S. Christiansen, A review of some integral equations for solving the Saint-Venant torsion problem, Journal of Elasticity (1978); 8 (1): 1-20.

S. Christiansen, P. C. Hansen, The e/ective condition number applied to error analysis of certain boundary collocation methods.J. Comp.Appl. Math. (1994); 54:15-36.

C. Constanda, The boundary integral equation method in plane elasticity, Proc. Am. Math. Soc. (1995);123 (11): 33853396.

C. Constanda, Inteqral equations of the rst kind in plane elasticity, Quart. Appl. Math. Mech. Solids (1995); 1: 251260.

G. Fairweather, A. Karageorghis, The method of fundamental solutions for elliptic boundary-value problems, Adv. Comput. Math. (1998); 9: 69-95.

M. Filonenko-Borodich, Theory of Elasticity, Dover Publications, New York, (1965). [31] J.A.T. Freitas, J.P.M. Almeida, Eds., Proc. 2-nd International Workshop: Tretz method, Computer Assisted Mechanics and Engineering Science (special issue) 8, n 2/3 (2001).

A. S. Gjam, H. A. Abdusalam, A. F. Ghaleb, Solution for a problem of linear plane elasticity with mixed boundary conditions on an ellipse by the method of boundary integrals, Journal of the Egyptian Mathematical Society (2013); 21: 361369.

W. C. Hassenp ug, Torsion of uniform bars with polygon cross-section, Computers and Mathematics with Applications (2003); 46: 313392.

J. Helsing, Integral equation methods for elliptic problems with boundary conditions of mixed type, J. Comp. Phys. (2009); 228: 88928907.

L. R. Hermann, Elastic torsional analysis of irregular shapes, Journal of the Engineering Mechanics Division, ASCE (1965); 91: 11-19.

I. Herrera, Boundary methods: A criterion for completeness, Proc. Natl. Acad. Sci. USA (1980); 77 (8): 4395-4398.

I. Herrera, Tretz-Herrera method, CAMES (1997); 4: 369 382.

I. Herrera, H. Gourgen, Boundary methods, C-complete systems for Stokes problems, Comput. Meth. appl. Mech. Engng. (1982); 30: 225-244.

D. L. Holl, E. W. Anderson, Limits of approximate solutions 
of a torsion problem, Bull. Am. Math. Soc. (1931); 37 (8): 580-584.

S. C. Huang, R. P. Shaw, The Tretz method as an integral equation. Adv. Engng. Software (1995); 24: 57-63.

M. A. Jaswon, A. R.S. Ponter, An integral equation solution of the torsion problem, Proc. Roy. Soc. London, Ser. A (1963); 1353: 237-246.

M. A. Jaswon, G.T. Symm, Integral equation methods in potential theory and elastostatics, Academic Press, London-New York-San Francisco (1977).

J. Jirousek, A. Wr blewski, T-elements: State of the art and future trends, Archives of Computational Methods in Engineering (1996); 3 (4): 323-434.

A. Karageorghis, The method of fundamental solutions for the calculation of the eigenvalues of the Helmholtz equation. Appl. Math. Lett. (2001); 14: 837-42.

M. Katsurada, U. Okamoto, The collocation points of the fundamental solution method for the potential problem. Computers and Mathematics with Applications (1996); 31(1): 123137.

J. T. Katsikadelis and E. J. Sapountzakis, Torsion of composite bars by boundary element method, J.Eng.Mech. (1985); 111:1197-1210.

Th. Kermanidis, Kupradze s functional equation for the torsion problem of prismatic bars-Part 1, Computer methods in applied mechanics and engineering (1976); 7: 39 46.

Th. Kermanidis, Kupradze s functional equation for the torsion problem of prismatic bars-Part 2, Computer methods in applied mechanics and engineering (1976); 7: 249259 .

E. Kita and N. Kamiya, Tretz method: An overview, Advances in Engineering Software (1995); 24: 3-12.

J. A. Ko odziej, A. Fraska, Elastic torsion of bars possessing regular polygon in crosssection using BCM, Computers and Structures (2005); 84: 7891.

J. A. Kolodziej, M. Kleiber, Boundary collocation method vs FEM for some harmonic 2-D problems, Comp. and Structures (1989); 33: 155-168.

V. D. Kupradze, Methods of potential in Theory of Elasticity, Fizmatgiz, Moscow (1963).

C. S. Liu, A meshless regularized integral equation method (MRIEM) for Laplace equation in arbitrary interior or exterior plane domains, ICCES (2007); 3 (2): 57-68.

Z. C. Li, Y. L. Chen, G. G. Georgiou, C. Xenophontos, Special boundary approximation methods for Laplace equation problems with boundary singularities-applications to the Motz problem, Inter. Comp. Math. Appl. (2006); 51: 115142.

Z.-C. Li, P. C. Chu, L. J. Young, M. G. Lee, Combined Tretz methods of particular and fundamental solutions for corner and crack singularity of linear elastostatics, Eng. Anal. Bound. Elem. (2010); 34: 632654.

Z.-C. Li, T.-T. Lu, H.-T. Huang, A. H.-D. Cheng, Trełz, collocation, and other boundary methods-A comparison, Num. Meth. for Partial Di/erential Equations (2007); 23 (1): 93144

C. C. Lo, F. W. Niedenfuhr, Singular integral equation solution for torsion, Proc. Amer. Soc. Civil Eng., Jour.
Engng. Mech. Div. 96 EM 4 (1970) 535542.

R. Mathon, R. L. Johnston, The approximate solution of elliptic boundary-value problems by fundamental solutions. SIAM Journal on Numerical Analysis (1977); 14(4): 638650.

D. Natroshvili, I. G. Stratis, S. Zazashvili, Boundary integral equation methods in the theory of elasticity of hemitropic materials: a brief review, J. Comp. Appl. Math. (2010); 234: 16221630.

A. R. S. Ponter, An integral equation solution of the inhomogeneous torsion problem, SIAM Jour. Appl. Math. (1966); 14: 819830.

A. Poullicas, A. Karageorghis, G. Georgiou, Method of fundamental solutions for harmonic and biharmonic boundary-value problems, Comput. Mech. (1998); 21: 416-423.

S. W. Reagan, W. D. Pilkey, Constrained torsion of prismatic bars, nite elements in analysis and design (2002); 38:909 919.

Z. Rui, H. Jin, L. Tao, Meshless local boundary integral equation method for 2Delastodynamic problems, Eng. Anal. Bound. Elem. (2006); 30: 391398.

A. S. Saada, Elasticity theory and applications, Pergamon Press Inc., New York, Toronto, Oxford, Sydney, Braunschweig (1974).

V. Sladek, J. Sladek, S. N. Atluri, R. Van Keer, Numerical integration of singularities in meshless implementation of local boundary integral equations, Computational Mechanics (2000); 25: 394-403.

J. Sladek, V. Sladek, R. Van Keer, Meshless local boundary integral equation method for 2D elastodynamic problems, Int. J. Numer. Meth. Engng. (2003); 57: 235249.

I. S. Sokolnikd, Mathematical theory of elasticity, McgrawHill Book Company, Inc., New York Toronto London (1956).

E. Tretz, Ein Gegenst ck zum ritzchen Verfahren, Proc. 2-nd Int. Congr. Appl. Mech., Zurich, 131-137 (1926).

S. Timoshenko, J. N. Goodier, Theory of Elasticity, 2nd ed., McGraw-Hill, New York, (1951).

F. Y. M. Wan, On torsion of prismatic bodies, Studies Appl. Math. (1993); 89: 63-94.

D. L. Young, K. H. Chen, C. W. Lee, Novel meshless method for solving the potential problems with arbitrary domain, Journal of Computational Physics (2005) ; 209: 290321.

A. P. Zielinski, Ed., Proceedings of the 1-st International Workshop: Tretz method, Computer Assisted Mechanics and Engineering Science (special issue) 4, n 3/4 (1997).

A. P. Zielinski, I. Herrera, Tretz method: Fitting boundary conditions, Int. J. Numer. Meth. Eng. (1987); 24: 871891.

A. P. Zielinski, M. Zyczkowski, The trigonometric contour series method in application to clamped plates of arbitrary contour, Bulletin Pol. Acad. Sci./Appl. Mech. (1981); 29: 159-176.

T. Zlatanovski, On the solution of St.Venant s torsion problem by singular boundary integral equations, Z. Angew. Math. Mech. ( 1991); 9: 331-340. 